\title{
A Flux-Conservative Finite Difference scheme for Anisotropic Bioelectric Problems
}

\author{
George C. Bourantas ${ }^{1}$, Benjamin F. Zwick ${ }^{1}$, Grand R. Joldes ${ }^{1}$, Adam Wittek ${ }^{1}$, Karol Miller ${ }^{1,2}$ \\ ${ }^{1}$ Intelligent Systems for Medicine Laboratory, School of Mechanical and Chemical Engineering, The University of Western Australia, \\ 35 Stirling Highway, Perth, WA 6009, Australia \\ ${ }^{2}$ School of Engineering and Information Technology, Murdoch University, 90 South St, Murdoch, Australia
}

\begin{abstract}
We present a flux-conservative finite difference (FCFD) scheme for solving inhomogeneous anisotropic bioelectric problems. The method applies directly on the raw medical image data without the need for sophisticated image analysis algorithms to define the interface between materials with different electrical conductivities. We demonstrate the accuracy of the method by comparison with analytical solution. Results for a patient-specific head model highlight the applicability of the method.
\end{abstract}

Keywords: Flux-Conservative Finite Difference, Anisotropic electrical conductivity, Epilepsy

\section{Introduction}

Epilepsy is a neurological condition of recurrent or unprovoked seizures that is thought to affect $1 \%$ of children [1]. Antiepileptic drugs serve as the primary treatment [2]. Treatment strategy relies on two key issues. First, the quality of life of an epileptic patient fails to improve until the permanent cessation of seizures. Second, one third of patients experience drug resistance [2,3]. Surgery to remove or alter the region of the brain where seizures originate is recommended to patients who fail to respond to antiepileptic drug therapy [4].

Around 100,000-500,000 patients in the United States of America with drug-resistant epilepsy are surgical candidates each year [2]. However, due to the high risk associated with the surgical procedure, less than $1 \%$ of patients are treated this way [2]. For surgical epileptic seizure management, there are two realistic options available: focal resection; or disconnection of the epileptogenic cortex [3]. Of these two options, only complete focal resection of the epileptic lesion offers the possibility of eliminating seizures.

Success of the surgical intervention depends on the ability to accurately identify the seizure onset zone (SOZ), which is to be resected. Intracranial electrodes help to identify the SOZ and map eloquent areas of the brain [6]. Currently, the clinical standard for identifying the SOZ are invasive electroencephalography (iEEG) grids and strips, or stereoEEG (sEEG) electrodes, deployed stereotactically through holes in the skull [5]. The iEEG or sEEG data recorded during the day is collected and manually interpreted by expert neurophysiologists to identify the electrode(s) most implicated in seizure onset.

Patients (usually young) unable to tolerate conscious cortical mapping for resection are candidates for intracranial electrode-mediated extra-operative mapping [3]. The aim of this mapping is to identify the epileptogenic zone. This zone, which is characterized by low-voltage, fast-current neuronal activity, represents the minimum amount of cortex that must be resected to eliminate seizures [7]. Magnetic resonance images (MRIs) are routinely used to determine the distribution of various tissue types throughout the brain. EEGs are used to localize the SOZ and the corresponding area of the brain, which is known as the eloquent cortex [8]. Following the initial MRI, patients undergo a craniotomy 
to implant intracranial EEG electrodes to the edges of the dura [3]. A low-resolution computed tomography (CT) scan is then used to locate the electrodes within the deformed brain [9].

Source localization of the epileptic zone can be enhanced using computational methods combined with the available imaging modalities. The pre-surgical planning capabilities for resection of the epileptogenic cortex will then be more accurate. Calculating the voltage distribution throughout a patient-specific head model is a key component of the forward problem of EEG source localization. The forward problem has been solved in previous studies using a preoperative brain model $[4,7,10-11]$. However, a more efficient method for computing the voltage terms is required for patient-specific applications and efficient implementation into the clinical workflow. Previous studies employed finite element methods or boundary element methods to localize the epileptogenic source [12-14]. These methods, however, are limited by their dependence on meshes that sufficiently capture the discontinuity of electrical conductivities between the differing media within the head [15]. Another issue with mesh-based methods is their reliance on pre-determined boundary positions at patient-specific conductivity interfaces within the cortex. Although a high-quality mesh will provide a simple solution to the forward problem, it requires an experienced analyst, thereby decreasing the practicality of implementing this technology into clinical practice.

In this study, we apply the flux-conservative finite difference (FCFD) method to numerically solve the forward problem of EEG source localization. The bioelectric problem is described by a set of partial differential equations. FCFD method discretizes these equations into a system of linear algebraic equations. The numerical solution of the linearized system determines the electric potential distribution throughout a patient-specific conducting volume (head model). The FCFD method applies to the rectangular grid of material properties extracted from patient data. This eliminates image segmentation and meshing that is required in mesh-based methods. The conductivity assigned to each node is used to form a system of linear equations that is then solved to compute the voltage term. We apply an anisotropic tensor for the electrical conductivity. We solve a simple problem with analytical solution to highlight the accuracy of the proposed scheme before applying it to a patient-specific head model of an epilepsy patient.

\section{Methods}

\subsection{Electromagnetic Modeling Using the Flux-Conservative Finite Difference Method}

\subsubsection{Governing equations}

Source localization applies a linear model, often called leadfield matrix, to correlate measured electrode voltages to their cerebral current sources. Computing the leadfield matrix requires the numerical solution of Maxwell's equations within the head (conducting medium). Since the frequencies employed for EEG are typically less than $100 \mathrm{~Hz}$, transient signals are negligible, and the quasi-static approximation can be employed [4]. Therefore, the relationship between current sources and the induced voltage field is given as:

$$
\boldsymbol{\nabla} \cdot(\overline{\overline{\boldsymbol{\sigma}}}(\boldsymbol{x}) \nabla \Phi(\boldsymbol{x}))=\boldsymbol{\nabla} \cdot \boldsymbol{J}(\boldsymbol{x})
$$

with $\Phi(\boldsymbol{x})$ being the voltage potential at location $\boldsymbol{x}$ in the spatial domain $\Omega, \overline{\overline{\boldsymbol{\sigma}}}(\boldsymbol{x})$ the spatially varying conductance of the volume, and $\boldsymbol{J}(\boldsymbol{x})$ the current source density at the nodes of the volume. The inhomogeneous conductivity tensor $\overline{\overline{\boldsymbol{\sigma}}}(\boldsymbol{x})$ can be represented by a $3 \times 3$ matrix as

$$
\overline{\overline{\boldsymbol{\sigma}}}(\boldsymbol{x})=\left[\begin{array}{lll}
\sigma_{x x} & \sigma_{x y} & \sigma_{x z} \\
\sigma_{y x} & \sigma_{y y} & \sigma_{y z} \\
\sigma_{z x} & \sigma_{z y} & \sigma_{z z}
\end{array}\right]
$$


while the left-hand side of Eq. (1) in its expanded form is given as

$$
\begin{aligned}
\boldsymbol{\nabla} \cdot(\overline{\overline{\boldsymbol{\sigma}}}(\boldsymbol{x}) \nabla \Phi(\boldsymbol{x}))= & \frac{\partial}{\partial x}\left(\sigma_{x x} \frac{\partial \Phi}{\partial x}+\sigma_{x y} \frac{\partial \Phi}{\partial \mathrm{y}}+\sigma_{x z} \frac{\partial \Phi}{\partial z}\right)+ \\
& \frac{\partial}{\partial y}\left(\sigma_{y x} \frac{\partial \Phi}{\partial x}+\sigma_{y y} \frac{\partial \Phi}{\partial y}+\sigma_{y z} \frac{\partial \Phi}{\partial z}\right)+ \\
& \frac{\partial}{\partial z}\left(\sigma_{z x} \frac{\partial \Phi}{\partial x}+\sigma_{z y} \frac{\partial \Phi}{\partial \mathrm{y}}+\sigma_{z z} \frac{\partial \Phi}{\partial z}\right)
\end{aligned}
$$

Using the Taylor series expansion and applying the flux-conservative finite difference scheme we can compute the spatial derivatives of Eq. (3). In the FCFD method, we can efficiently and accurately deal with the anisotropy and the discontinuities in the electrical conductance of the different materials (e.g. bone, soft tissue) in the brain. In the FCFD method we do not apply the chain rule in the computation of the spatial derivatives in Eq. (3), instead we treat the terms in the parenthesis for the spatial derivatives $\frac{\partial}{\partial x}, \frac{\partial}{\partial y}, \frac{\partial}{\partial z}$ as the unknow field functions. Therefore, the typical methodology applied in the classical FD methods is extended to account for the anisotropy of the field variables.

\subsubsection{Flux-Conservative Finite Difference Method}

The FD method works efficiently on Cartesian grids (that can be directly obtained from DICOM images) and computes the nonlinear convective term $\boldsymbol{\nabla} \cdot(\overline{\overline{\boldsymbol{\sigma}}}(\boldsymbol{x}) \nabla \Phi(\boldsymbol{x}))$ by applying a flux-conservative scheme. All Flux-Conservative FD formulations give a nodal equation for the potential field $\Phi(\boldsymbol{x})$ at each node of the grid. The nodal equations finally form a linear algebraic system which can be solved using direct or iterative solvers (for FD method several robust solvers exist).

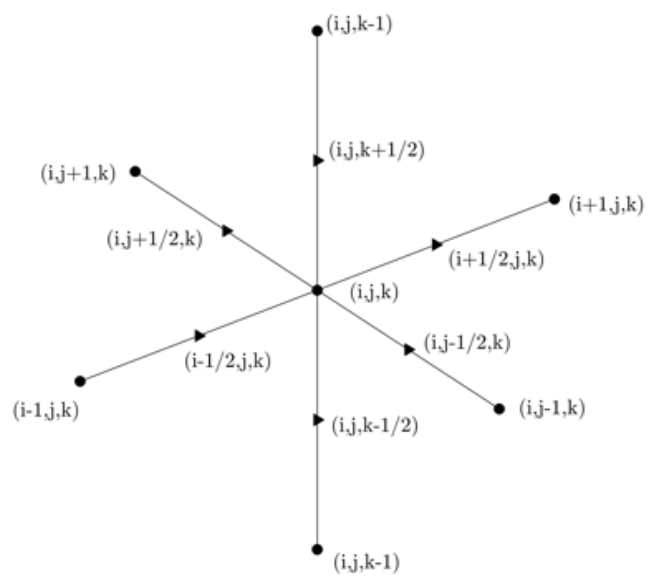

Fig. 1. The 3D stencil configuration used in the flux-conservative finite difference method.

This scheme computes spatial derivatives for the electric field using the stencil defined in Fig. 1. This is identical to the classical FD stencil except that in the FCFD stencil, fluxes in the fictitious grid points $((i+1 / 2, j),(i-1 / 2, j),(i, j+1 / 2)$, $(i, j+1 / 2))$ are preserved. Computation of the diffusion term at the grid points $((i, j),(i-1, j),(i, j+1),(i, j+1),(i, j-1))$ will lead to an erroneous non-conservative FD formulation. Application of classical (non-conservative) FD stencil by directly applying the chain rule to compute the spatial derivatives of the convective term will lead to incorrect calculation of fluxes. 
Using the flux conservative approach, the terms at the central node $(i, j, k)$ of the stencil shown in Fig. 1 can be written (for the $x$ coordinate) as

$\frac{\partial Q^{x}}{\partial x}=\frac{Q_{\left(1+\frac{1}{2}, j, k\right)}^{x}-Q_{\left(1-\frac{1}{2}, j, k\right)}^{x}}{h_{x}}$

where

$Q^{x}=\sigma_{x x} \frac{\partial \Phi}{\partial x}+\sigma_{x y} \frac{\partial \Phi}{\partial y}+\sigma_{x z} \frac{\partial \Phi}{\partial z}$

We compute the terms $\sigma_{x x}, \Phi_{, x}, \sigma_{x y}, \Phi_{, y}, \sigma_{x z}$ and $\Phi_{, z}$ on the off-grid nodes $\left(1+\frac{1}{2}, j, k\right)$ and $\left(1-\frac{1}{2}, j, k\right)$. The electrical conductance $\sigma_{x x}, \sigma_{x y}, \sigma_{x z}$ values are not defined on the off-grid nodes. Instead, they are computed using interpolating/approximating methods such as arithmetic averaging of the known values for the electrical conductance on the grid nodes, or the harmonic average. The former applies for the case of the $\sigma_{x x}$ electrical conductance (the same applies for $\sigma_{x y}$ and $\sigma_{x z}$ ) as

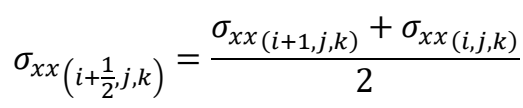

while the latter is written as

$\sigma_{x x_{\left(i+\frac{1}{2}, j, k\right)}}=\frac{2 \sigma_{x x_{(i+1, j)}} \sigma_{x x_{(i, j)}}}{\sigma_{x x_{(i+1, j)}}+\sigma_{x x}}$

The two approaches, despite their success in delivering reliable results, may result in decreased accuracy for the numerical solution when steep gradients in material properties (higher than 6 orders of magnitude) are present. This is because only the two nodes adjacent to the fictitious point are used in the computation, disregarding all the other nodes in the close vicinity. High order methods can be used to provide more accurate results but these increase the computational cost.

Furthermore, we need to compute the spatial derivatives of the electrical potential $\Phi(\boldsymbol{x})$ on the off-grid nodes. The derivative $\Phi_{, x}$ on the $\left(1+\frac{1}{2}, j, k\right)$ and $\left(1-\frac{1}{2}, j, k\right)$ nodes is given as

$\frac{\partial \Phi_{(i+1 / 2, j, k)}}{\partial x}=\frac{\Phi_{(i+1, j, k)}-\Phi_{(i, j, k)}}{h_{x}}$

and

$\frac{\partial \Phi_{(i-1 / 2, j, k)}}{\partial x}=\frac{\Phi_{(i, j, k)}-\Phi_{(i-1, j, k)}}{h_{x}}$

The derivative $\Phi_{, y}$ on the $\left(1+\frac{1}{2}, j, k\right)$ and $\left(1-\frac{1}{2}, j, k\right)$ nodes is given as 
$\frac{\partial \Phi_{(i+1 / 2, j, k)}}{\partial y}=\frac{\Phi_{\left(i+\frac{1}{2}, j+1 / 2, k\right)}-\Phi_{\left(i+\frac{1}{2}, j-1 / 2, k\right)}}{h_{y}}$

and

$\frac{\partial \Phi_{(i-1 / 2, j, k)}}{\partial y}=\frac{\Phi_{\left(i-\frac{1}{2}, j+1 / 2, k\right)}-\Phi_{\left(i-\frac{1}{2}, j-1 / 2, k\right)}}{h_{y}}$

where

$\Phi_{\left(i+\frac{1}{2}, j+1 / 2, k\right)}=\frac{\Phi_{(i, j, k)}+\Phi_{(i+1, j, k)}+\Phi_{(i+1, j+1, k)}+\Phi_{(i, j+1, k)}}{4}$

$\Phi_{\left(i+\frac{1}{2}, j-1 / 2, k\right)}=\frac{\Phi_{(i, j, k)}+\Phi_{(i+1, j, k)}+\Phi_{(i+1, j-1, k)}+\Phi_{(i, j-1, k)}}{4}$

$\Phi_{\left(i-\frac{1}{2}, j+1 / 2, k\right)}=\frac{\Phi_{(i, j, k)}+\Phi_{(i, j+1, k)}+\Phi_{(i-1, j+1, k)}+\Phi_{(i-1, j, k)}}{4}$

$\Phi_{\left(i-\frac{1}{2}, j-1 / 2, k\right)}=\frac{\Phi_{(i, j, k)}+\Phi_{(i, j-1, k)}+\Phi_{(i-1, j, k)}+\Phi_{(i-1, j-1, k)}}{4}$

Finally, the derivative $\Phi_{, z}$ on the $\left(1+\frac{1}{2}, j, k\right)$ and $\left(1-\frac{1}{2}, j, k\right)$ nodes is given as

$\frac{\partial \Phi_{(i+1 / 2, j, k)}}{\partial z}=\frac{\Phi_{\left(i+\frac{1}{2}, j+\frac{1}{2}, k+1 / 2\right)}-\Phi_{\left(i+\frac{1}{2}, j-\frac{1}{2}, k-1 / 2\right)}}{h_{z}}$

and

$\frac{\partial \Phi_{(i-1 / 2, j, k)}}{\partial z}=\frac{\Phi_{\left(i-\frac{1}{2}, j+\frac{1}{2}, k+1 / 2\right)}-\Phi_{\left(i-\frac{1}{2}, j-\frac{1}{2}, k-1 / 2\right)}}{h_{z}}$

where

$\Phi_{\left(i+\frac{1}{2}, j+1 / 2, k\right)}=\frac{\Phi_{(i, j, k)}+\Phi_{(i+1, j, k)}+\Phi_{(i+1, j+1, k)}+\Phi_{(i, j+1, k)}}{4}$

Consequently, for computing the partial derivative with respect to $x$ for the $Q^{x}$ term, nine neighbors are involved. Figure 2 shows the grid nodes used in the computation of the term $\frac{\partial}{\partial x}\left(\sigma_{x x} \frac{\partial \Phi}{\partial x}+\sigma_{x y} \frac{\partial \Phi}{\partial y}+\sigma_{x z} \frac{\partial \Phi}{\partial z}\right)$. 

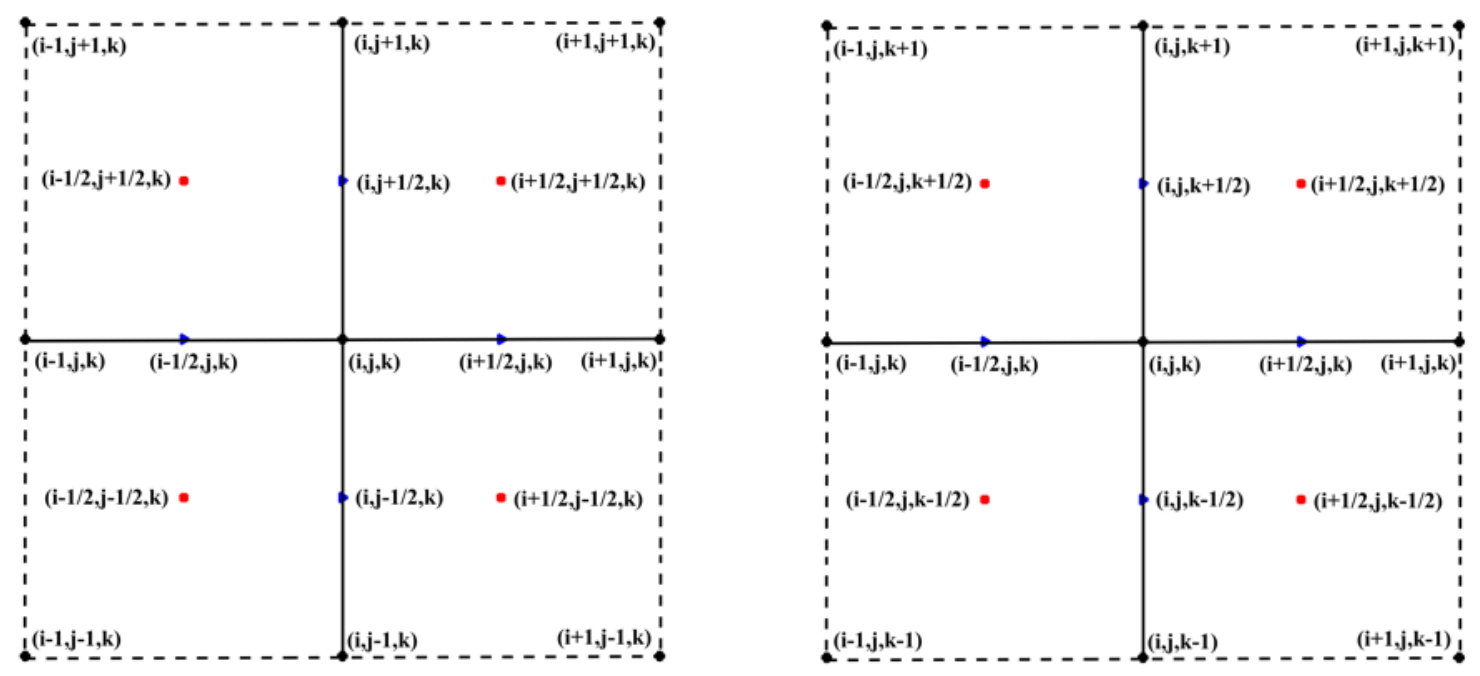

Fig. 2. The 3D stencil configuration used in the flux-conservative finite difference method.

The same procedure applies for the other two partial derivatives $\frac{\partial}{\partial y}\left(\sigma_{y x} \frac{\partial \Phi}{\partial x}+\sigma_{y y} \frac{\partial \Phi}{\partial y}+\sigma_{y z} \frac{\partial \Phi}{\partial z}\right)$ and $\frac{\partial}{\partial z}\left(\sigma_{z x} \frac{\partial \Phi}{\partial x}+\right.$ $\left.\sigma_{z y} \frac{\partial \Phi}{\partial \mathrm{y}}+\sigma_{z z} \frac{\partial \Phi}{\partial z}\right)$ in Eq. (3). Therefore, twenty-seven neighboring nodes form the stencil for computing the left-hand side in Eq. (1). The right-hand side $(\boldsymbol{\nabla} \cdot \boldsymbol{J}(\boldsymbol{x}))$ is also defined on the grid nodes and can be defined as a continuous function, discretized over the nodes, or as point sources.

\section{Results}

\subsection{Verification of the FCFD scheme}

To demonstrate the accuracy of the proposed FCFD scheme we solve the Laplace equation for an inhomogeneous anisotropic medium in a unit volume box. The problem has an analytical solution of the form

$\Phi(\boldsymbol{x})=e^{x+y+z}$

For an inhomogeneous anisotropic medium, the conductivity tensor of the analytical solution has the form

$\overline{\overline{\boldsymbol{\sigma}}}(\boldsymbol{x})=\left[\begin{array}{ccc}e^{x+y+z} & -0.25 e^{x+y+z} & -0.75 e^{x+y+z} \\ -0.25 e^{x+y+z} & 1.5 e^{x+y+z} & -1.25 e^{x+y+z} \\ -0.75 e^{x+y+z} & -1.25 e^{x+y+z} & 2 e^{x+y+z}\end{array}\right]$

We apply Dirichlet boundary conditions on the boundary nodes, according to the analytical solution (Eq. 19).

The linear system of the Laplace equation can be solved using direct or iterative solvers. The former are extremely accurate but have memory limitations, especially for 3D problems with large number of nodes. The latter do not always converge but are extremely efficient and have less computational cost compared to direct solvers. For the systems used in the present study, we use the minimum residual method, which applies to nonsymmetric systems. We used an Intel i7 quad core processor with 16 GB RAM for our simulations. 
We compare the numerical solution against the analytical one using the Normalized Root Mean Square Error defined as NRMSE $=\frac{\sqrt{\frac{1}{N} \sum_{i=1}^{N}\left(u_{i}^{\text {numerical }}-u_{i}^{\text {analytical }}\right)^{2}}}{u_{\text {max }}^{\text {analytical }}-u_{\text {min }}^{\text {analytical }}}$. To study the convergence of the solution, we used successively denser grids starting from $51 \times 51 \times 51$ up to $201 \times 201 \times 201$.

Table 1. Maximum relative error and normalized root mean square error (NRMSE) for increasing grid resolution.

\begin{tabular}{cccc}
\hline Grid resolution & Solution time (s) & $\boldsymbol{L}_{\infty}$ & NRMSE \\
\hline $51 \times 51 \times 51$ & 13 & $2.21 \times 10^{-5}$ & $2.13 \times 10^{-6}$ \\
$101 \times 101 \times 101$ & 228 & $1.02 \times 10^{-5}$ & $7.08 \times 10^{-7}$ \\
$201 \times 201 \times 201$ & 3363 & $6.72 \times 10^{-6}$ & $6.59 \times 10^{-7}$ \\
\hline
\end{tabular}

The results (Table 1) suggest that both the maximum relative error and NRMSE will converge to zero as the number of nodes increases, confirming the accuracy of the FCFD scheme for solving anisotropic, three-dimensional, bioelectric field problems. Figure 3 shows the potential distribution computed by the analytical solution at plane $\mathrm{z}=0.5$ and a histogram displaying the differences, node by node, of the numerical solution with the analytical one for a grid resolution of $101^{3}$.

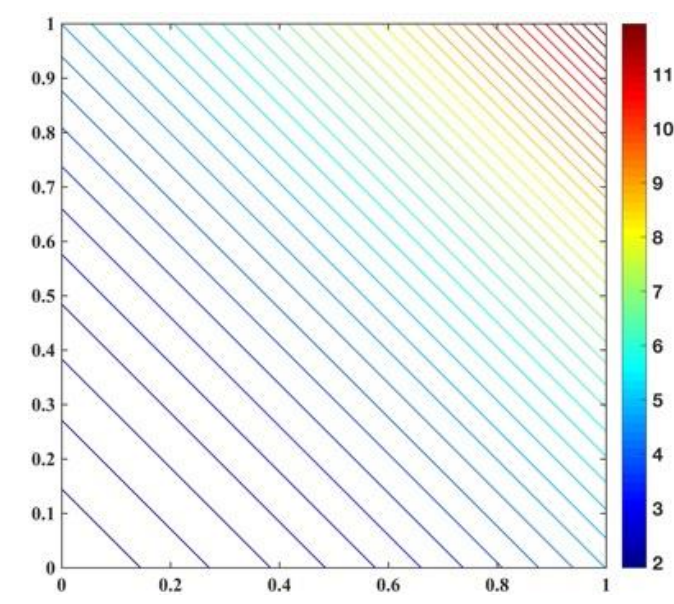

(a)

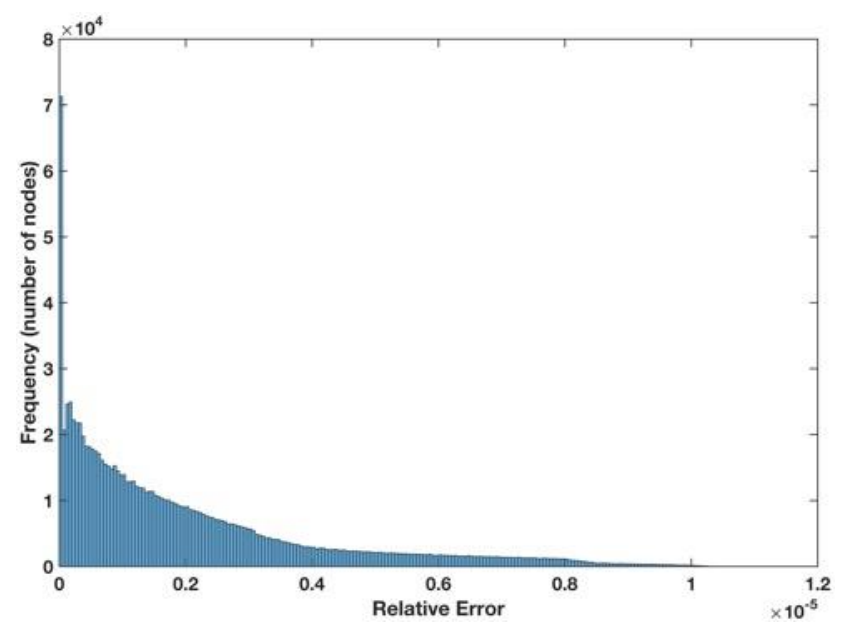

(b)

Fig. 3. Axial view of the (a) numerical solution and (b) histogram of the differences with the analytical solution using the fluxconservative finite difference method for the for inhomogeneous anisotropic medium verification problem.

For source localization, the computational time needed to solve the forward problem is crucial because multiple forward problems must be solved. Therefore, the accuracy and efficiency provided from the proposed scheme makes it a strong candidate to be used in clinical practice.

\subsection{Patient-specific head model}

In this section, we apply the FCFD method to a patient-specific head model of a five-year old epilepsy patient. The electrical conductivities were extracted from the patient's diffusion-weighted MRI using the method described in [16]. A node was assigned to the corner of each voxel to create a $160 \times 192 \times 192$ grid comprised of 5,898,240 points. An isotropic conductivity was assigned to all nodes inside the conducting volume. The three-dimensional finite difference brain volume was comprised of white and grey matter, as well as cerebrospinal fluid and air. We model air, grey matter and cerebrospinal fluid conductivities using isotropic tensors, while white matter fibers were assigned anisotropic tensors. Using the Cartesian grid (voxels) directly from the raw data we avoid the need for image segmentation to assign material properties. 


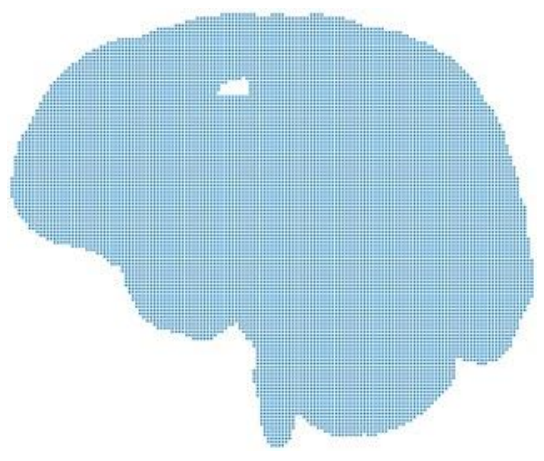

(a)

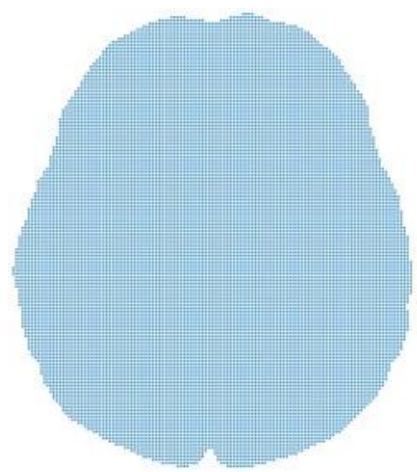

(b)

Fig. 4. (a) Sagittal and (b) axial view of the brain raw data

We compute the electric potential distribution throughout the brain volume by applying the point electrode model. We selected electrodes as the source and sink. We apply a current of $1 \mathrm{~A}$ at the source, and we remove $1 \mathrm{~A}$ at the sink. In the presence of any external current source, Poisson's equation (Equation 10) governs the potential distribution within the head volume incorporating anisotropic conductivity. At the boundaries, we enforce Neumann boundary conditions (Equation 2). We numerically solve the linear system equations using the minimum residual method. We model air using an isotropic conductivity of $10^{-9}$, assigning this to all voxels outside of the head volume. This is demonstrated in Figures 5(a)-(c) as the voltage approaches zero outside the skull-air interface boundary.

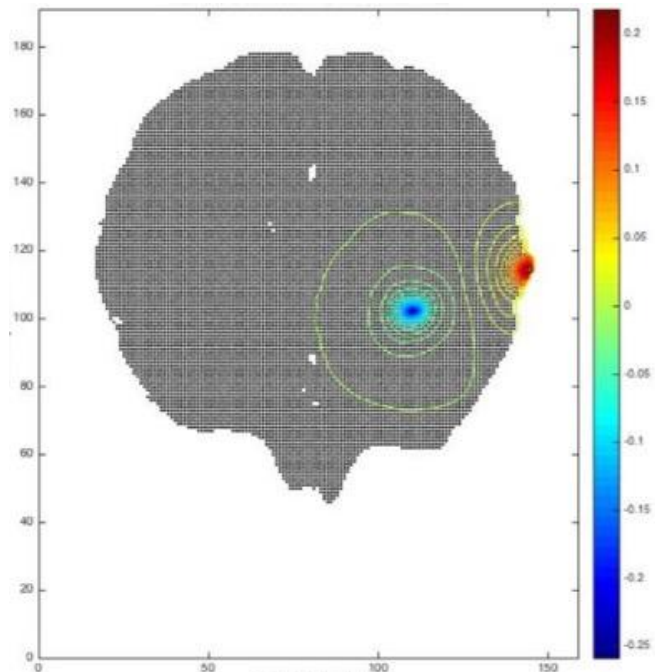

(a)

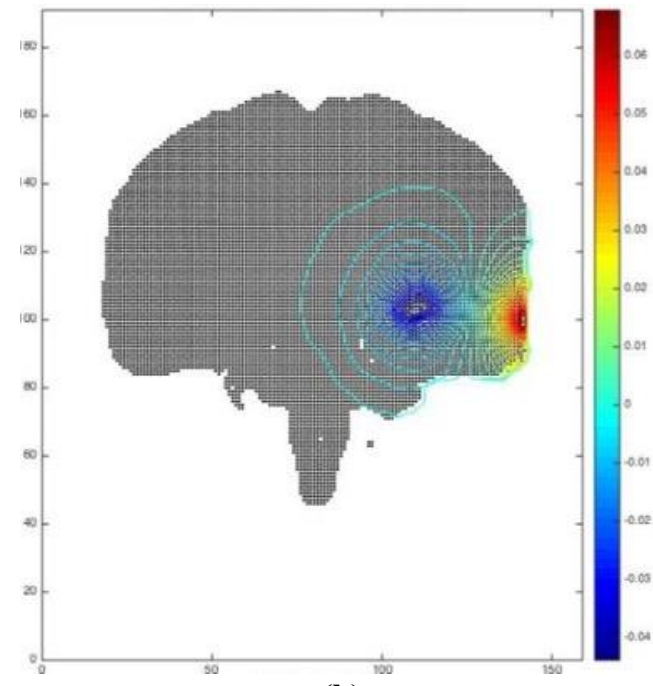

(b) 


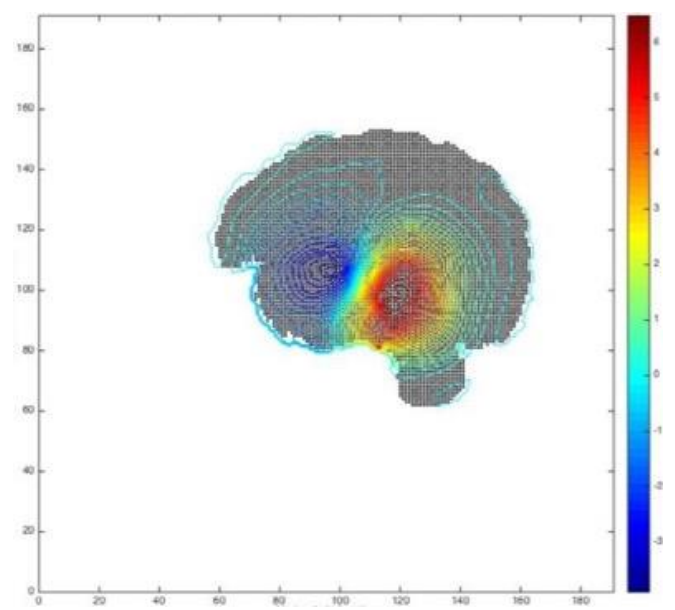

(c)

Fig. 5. Electric potential distribution throughout the brain in (a) axial plane 103, (b) coronal plane 108, and (c) sagittal plane 127.

Figure 5 shows the electric potential distribution throughout the brain in the axial, coronal and sagittal planes. These slices center around the midpoint of the preselected source/sink configuration to best illustrate the voltage distribution (we positioned the source at $(143,114,101)$ and the sink at $(110,102,104)$ ). As expected, the source and sink generate a voltage inside the conducting volume that is greatest close to the corresponding electrodes and approaches zero as the distance from these regions increases.

\section{Conclusion}

In this study, we successfully applied the FCFD method to numerically solve the bioelectric problem to obtain the voltage distribution throughout the head. We first applied the FCFD method to a simple problem with an analytic solution. Following verification, the proposed scheme has been applied to a patient-specific head model (created using raw medical image data) to compute the electric potential distribution throughout the conducting volume for a specified source/sink configuration.

The accuracy of the patient-specific head model may be improved by using a complete electrode model instead of the point-electrode model used in the present study. The complete electrode model incorporates the size of the electrodes, their shape and the contact impedance, providing a better approximation of the electrode-tissue interface. With the point-electrode model, currents in the electrodes are not considered in the numerical solution. Therefore, the voltages close to the electrodes are of greater amplitude compared to those expected in real-world cases.

Successful application of the proposed scheme enhances current pre-surgical planning capabilities for resection of the epileptogenic cortex. In contrast to traditional mesh-based methods such as the finite element and boundary element methods, with our method there is no need for image segmentation and mesh generation.

\section{Acknowledgements}

This research was supported by the Australian Government through the Australian Research Council's Discovery Projects funding scheme (project DP160100714) and National Health and Medical Research Council Project Grant APP1162030. 


\section{References}

1. Devinsky, O.: Epilepsy: A patient and family guide, Demos Medical Publishing, New York (2007)

2. Ravindra, VM, Sweney, MT, Bollo, RJ: 'Recent developments in the surgical management of paediatric epilepsy', Archives of Disease in Childhood, vol. 102, no. 8, pp. 760-776 (2017).

3. Husain, AMMD Practical Epilepsy, 1st ed. edn, Springer Publishing Company, New York (2015).

4. Hyde, DE, Tomas-Fernandez, X, Stone, SS, Peters, J \& Warfield, SK 2017, 'Localization of stereoelectroencephalography signals using a finite difference complete electrode model', in Engineering in Medicine and Biology Society (EMBC), 2017 39th Annual International Conference of the IEEE, IEEE, pp. 3600-3603.

5. Vega, R.A., Holloway, K.L., Larson, P.S.: Image-Guided Deep Brain Stimulation. Neurosurg Clin N Am 25, 159172 (2014)

6. Hallez, H, Vanrumste, B, Grech, R, Muscat, J, De Clercq, W, Vergult, A, D'Asseler, Y, Camilleri, KP, Fabri, SG, et al. 2007, 'Review on solving the forward problem in EEG source analysis', Journal of NeuroEngineering and Rehabilitation, vol. 4, no. 1, pp. 46-75.

7. David, O, Blauwblomme, T, Job, A-S, Chabardès, S, Hoffmann, D, Minotti, L \& Kahane, P 2011, 'Imaging the seizure onset zone with stereo-electroencephalography', Brain: A Journal of Neurology, vol. 134, no. 10, pp. 2898-2911.

8. Taimouri, V, Akhondi-Asl, A, Tomas-Fernandez, X, Peters, JM, Prabhu, SP, Poduri, A, Takeoka, M, Loddenkemper, T, Bergin, AMR, et al. 2014, 'Electrode localization for planning surgical resection of the epileptogenic zone in pediatric epilepsy', International journal of computer assisted radiology and surgery, vol. 9, no. 1, pp. 91-105.

9. Hermes, D, Miller, KJ, Noordmans, HJ, Vansteensel, MJ \& Ramsey, NF 2010, 'Automated electrocorticographic electrode localization on individually rendered brain surfaces', Journal of neuroscience methods, vol. 185, no. 2, pp. 293-298.

10. Brodbeck, V, Spinelli, L, Lascano, AM, Wissmeier, M, Vargas, M-I, Vulliemoz, S, Pollo, C, Schaller, K, Michel, CM, et al. 2011, 'Electroencephalographic source imaging: a prospective study of 152 operated epileptic patients', Brain, vol. 134, no. 10, pp. 2887-2897.

11. Erem, B, Hyde, DE, Peters, JM, Duffy, FH \& Warfield, SK 2017, 'Dynamic electrical source imaging (desi) of seizures and interictal epileptic discharges without ensemble averaging', IEEE transactions on medical imaging, vol. 36, no. 1, pp. 98-110.

12. Dannhauer, M, Lanfer, B, Wolters, CH \& Knösche, TR 2011, 'Modeling of the human skull in EEG source analysis', Human brain mapping, vol. 32, no. 9, pp. 1383-1399.

13. Pursiainen, S, Lew, S \& Wolters, CH 2016, 'Forward and inverse effects of the complete electrode model in neonatal EEG', Journal of neurophysiology, vol. 117, no. 3, pp. 876-884.

14. Rullmann, M, Anwander, A, Dannhauer, M, Warfield, SK, Duffy, FH \& Wolters, CH 2009, 'EEG source analysis of epileptiform activity using a $1 \mathrm{~mm}$ anisotropic hexahedra finite element head model', Neurolmage, vol. 44, no. 2, pp. 399-410.

15. Hédou-Rouillier, V 2004, 'A finite difference method to solve the forward problem in electroencephalography (EEG)', Journal of Computational and Applied Mathematics, vol. 167, no. 1, pp. 35-58.

16. Tuch, DS, Wedeen, VJ, Dale, AM, George, JS \& Belliveau, JW 2001, 'Conductivity tensor mapping of the human brain using diffusion tensor MRI', Proceedings of the National Academy of Sciences, vol. 98, no. 20, pp. 1169711701. 